\title{
Association schemes, fusion rings, C-algebras, and reality-based algebras where all nontrivial multiplicities are equal
}

\author{
Harvey I. Blau
}

Received: 8 June 2009 / Accepted: 23 July 2009 / Published online: 18 August 2009

(C) Springer Science+Business Media, LLC 2009

\begin{abstract}
Multiplicities corresponding to irreducible characters are defined for reality-based algebras. These algebras with a distinguished basis include fusion rings, $\mathrm{C}$-algebras, and the adjacency algebras of finite association schemes. The definition of multiplicity generalizes that for schemes. For a broad class of these structures, which includes the adjacency algebras, it is proved that if all the nontrivial multiplicities are equal then the algebra is commutative, and is a $\mathrm{C}$-algebra if its dimension is larger than two.
\end{abstract}

Keywords Reality-based algebra $\cdot \mathrm{C}$-algebra $\cdot$ Adjacency algebra $\cdot$ Association scheme $\cdot$ Fusion ring $\cdot$ Multiplicity

\section{Introduction}

This article contains a result, for a fairly general class of algebras with distinguished basis, that has the following consequence: an association scheme all of whose nontrivial multiplicities are equal must be commutative. In the proof [11] of their theorem that an association scheme of prime order is commutative, Hanaki and Uno show that all the nonprincipal irreducible characters of the adjacency algebra of the scheme are algebraically conjugate; hence that the nontrivial multiplicities are equal. They then cite the Bannai-Ito argument [4, Theorem II.4.3] to obtain that all the nontrivial valencies are equal, and reach their conclusion via the theorem of Arad, Fisman, and Muzychuk [2, Theorem 1.2]. So our result shortens this proof, and our derivation yields the Bannai-Ito result along the way. It also applies to fusion rings.

The author has learned from one of the referee's reports on the originally submitted version of this article, that the present result, in the case of adjacency algebras of association schemes, was discovered earlier by Hanaki and Uno. It appears

H.I. Blau $(\bowtie)$

Department of Mathematical Sciences, Northern Illinois University, DeKalb, IL 60115, USA

e-mail: blau@math.niu.edu 
on Hanaki's website (http://math.shinshu-u.ac.jp/ hanaki) as an unpublished note remarking on [11]. It is precisely our Corollary 2 below. Our main theorem (Theorem 1 below) has more general hypotheses and a proof that is somewhat different from theirs. In particular, we invoke neither the Frame number nor the arithmetic mean/geometric mean inequality.

Some definitions and notation are needed to establish the context for the main theorem.

Definition 1 [6, Definition 1.16] A reality-based algebra $(\mathrm{RBA})(A, B)$ is an algebra $A$ over $\mathbb{C}$ with a distinguished basis $B=\left\{b_{i} \mid 0 \leq i \leq d\right\}$, where $d<\infty, b_{0}=1_{A}$, and the following three conditions hold:

(1a) For all $0 \leq i, j \leq d$,

$$
b_{i} b_{j}=\sum_{l=0}^{d} \beta_{i j l} b_{l},
$$

where each coefficient (structure constant) $\beta_{i j l}$ is in $\mathbb{R}$.

(1b) There is an algebra anti-automorphism * of $A$, such that $\left(^{*}\right)^{2}=i d_{A}$ and $B^{*}=B$. (So ${ }^{*}$ has order at most two, and permutes the elements of $B$. Set $b_{i^{*}}:=b_{i}{ }^{*}$.)

(1c) For all $0 \leq i, j \leq d$,

$$
\beta_{i j 0}=0 \text { if } j \neq i^{*} \text {, and } \beta_{i i^{*} 0}=\beta_{i^{*} i 0}>0 .
$$

Reality-based algebras include C-algebras that are both commutative $[4,5,13]$ and noncommutative [8], table algebras that are both commutative [1] and noncommutative [2, 7, 10], and hypergroups as in [14]. The adjacency algebra, or Bose-Mesner algebra, of an association scheme is an example of a RBA. That is, given an association scheme (in the sense of [15]) on an underlying set with $n<\infty$ elements, each relation of the scheme is encoded in an $n \times n 0 / 1$ matrix. The set of these $a d-$ jacency matrices forms a basis for the algebra that it generates, and Definition 1 is satisfied, where the anti-automorphism is matrix transpose. The structure constants for an adjacency algebra are necessarily nonnegative integers. A fusion ring [9, Definition 2.1] is a RBA where all the structure constants $\beta_{i j l}$ are nonnegative integers, and all $\beta_{i i^{*} 0}=1$.

Definition 2 [6, Definitions 1.1d, 2.10] A degree map for a $\operatorname{RBA}(A, B)$ is an algebra homomorphism $\delta: A \rightarrow \mathbb{C}$ such that $\delta\left(b_{i}\right) \in \mathbb{R} \backslash\{0\}$ for $0 \leq i \leq d$. The values $\delta\left(b_{i}\right)$ are called the degrees of $(A, B, \delta)$. A degree map $\delta$ is positive if $\delta\left(b_{i}\right)>0$ for $0 \leq i \leq d$. If $\delta$ is a degree map such that $\delta\left(b_{i}\right)=\beta_{i i *}$ for all $i$, then $\delta$ (or the triple $(A, B, \delta))$ is called standard.

For any degree map $\delta, \delta\left(b_{i}\right)=\delta\left(b_{i}{ }^{*}\right)$ for all $i$ (see Corollary 5 below). A given RBA may have no degree map [6, Example 1.1] or several (e.g., ( $\mathbb{C} G, G)$ where $G$ is the symmetric group $S_{n}$ and $\delta$ is either the principal character or the sign character). A RBA has at most one positive degree map (see Theorem 2 below) and indeed has a positive degree map whenever all the structure constants are nonnegative (see Theorem 3 below). The adjacency algebra of an association scheme has a standard 
degree map $\delta$, where $\delta\left(b_{i}\right)$ is the row sum of the $0 / 1$ matrix $b_{i}$, that is, the valency of the corresponding relation.

Definition 3 [7, Definition 1.6], [6, Definitions 2.12, 2.13] Let $(A, B)$ be a RBA with given degree map $\delta$. Then the stable degree map $\sigma: B \rightarrow \mathbb{R} \backslash\{0\}$ is defined by

$$
\sigma\left(b_{i}\right):=\delta\left(b_{i}\right)^{2} / b_{i i^{*} 0}, 0 \leq i \leq d .
$$

The order of $B$ (actually, of $(B, \delta)$ ), is defined by

$$
o(B):=\sum_{i=0}^{d} \sigma\left(b_{i}\right) .
$$

Note that if $\delta$ is standard, then $\delta=\sigma$. In particular, if $(A, B)$ is the adjacency algebra of an association scheme, then $o(B)$ is the sum of the valencies; that is, the cardinality of the underlying set of the scheme.

The basis $B$ of a RBA can be rescaled [1, Section 2], [6, Definition 2.11], replacing each $b_{i}$ by $\lambda_{i} b_{i}$ for some $\lambda_{i} \in \mathbb{R} \backslash\{0\}$ such that $\lambda_{i}=\lambda_{i}$ for all $i$ and $\lambda_{0}=1$. It is easily seen that the rescaled basis again yields a RBA. The degrees and structure constants will usually change under rescaling, but the stable degrees, and hence the order, do not. If a degree $\delta\left(b_{i}\right)$ is negative, rescaling $b_{i}$ and $b_{i *}$ to $-b_{i}$ and $-b_{i}$ resp. yields positive degrees.

Definition $4 \mathrm{~A}$ degree map $\delta$ of a $\operatorname{RBA}(A, B)$ is a full degree map if $\delta\left(b_{i}\right) \geq \beta_{i i^{*} 0}$ for $0 \leq i \leq d$.

If $\delta$ is standard then trivially it is a full degree map. If $(A, B)$ is a fusion ring then there is a unique positive degree map $\delta$ (Theorem 3 below). In this case for a fusion ring,

$$
\begin{aligned}
\delta\left(b_{i}\right)^{2}=\delta\left(b_{i}\right) \delta\left(b_{i}{ }^{*}\right)=\delta\left(b_{i} b_{i}^{*}\right) & =\delta\left(1 \cdot b_{0}+\sum_{l=1}^{d} \beta_{i i^{*} l} b_{l}\right) \\
& =1+\sum_{l=1}^{d} \beta_{i i^{*} l} \delta\left(b_{l}\right) \geq 1
\end{aligned}
$$

So $\delta\left(b_{i}\right) \geq 1=\beta_{i i^{*} 0}$, and $\delta$ is a full degree map. Furthermore, if $\delta\left(b_{i}\right)=1$ for all $i$ then $B$ is a group.

Every reality-based algebra $(A, B)$ is semisimple [6, Proposition 2.3], [12, Section 5]. So the irreducible characters $\chi_{s}$ of $A$ are in bijection with the primitive central idempotents $e_{s}$ of $A, 1 \leq s \leq k: A=\oplus_{s=1}^{k} e_{s} A ; e_{s} A \cong M_{n_{s}}(\mathbb{C})$, a full matrix algebra of degree $n_{s}\left(n_{s} \in \mathbb{Z}_{>0}\right) ; \chi_{s}\left(e_{t} A\right)=0$ if $s \neq t$, and $\chi_{s}\left(e_{s}\right)=\chi_{s}(1)=n_{s}$. Thus, $d+1=\operatorname{dim} A=\sum_{s=1}^{k} n_{s}{ }^{2}$, and $A$ is commutative if and only if $n_{s}=1$ for $1 \leq s \leq k$. Since any degree map is an irreducible character, when there is a degree map $\delta$ that we distinguish, we denote it as $\delta=\chi_{1}$. 
Definition 5 Let $(A, B)$ be a RBA. A feasible trace function $[2,12]$ is a linear map $\phi: A \rightarrow \mathbb{C}$ such that $\phi(x y)=\phi(y x)$ for all $x, y \in A$.

Since $A$ is semisimple and since the only feasible trace functions on a full matrix algebra are scalar multiples of the ordinary trace, it follows that any feasible trace function on $A$ is a linear combination of the irreducible characters $\chi_{s}$.

Definition 6 [3, Section 3] Let $(A, B)$ be a RBA with degree map $\delta=\chi_{1}$ and order $o(B)$ (with resp. to $\delta$ ). The standard feasible trace function is the linear map $\tau: A \rightarrow \mathbb{C}$ such that $\tau\left(b_{0}\right)=o(B)$ and $\tau\left(b_{i}\right)=0$, all $1 \leq i \leq d$.

It follows from Definition $1 \mathrm{c}$ that $\tau$ is indeed a feasible trace map.

Definition 7 Let $(A, B)$ be a RBA with degree map $\delta=\chi_{1}$ and consequent standard feasible trace $\tau$. The multiplicities $m_{s}, 1 \leq s \leq k$, for $\left(A, B, \delta=\chi_{1}\right)$ are defined as the coefficients in the equation

$$
\tau=\sum_{s=1}^{k} m_{s} \chi_{s}
$$

The multiplicities are positive real numbers, and $m_{1}=1$ (see Proposition 1 and Corollary 6 below). As is well known, the feasible trace function for the adjacency algebra of an association scheme is the character of the standard module, and the $m_{s}$ are positive integers. Note that the irreducible characters, standard feasible trace function, and multiplicities of a RBA are not affected by rescaling the distinguished basis.

Now we can state the main theorem.

Theorem 1 Suppose that $(A, B)$ is a RBA with a full degree map $\delta=\chi_{1}$. Suppose as well that all the structure constants $\beta_{i j l}$ are integers. If the multiplicities $m_{s}$ are equal for all $2 \leq s \leq k$, then $A$ is commutative and the degrees $\chi_{1}\left(b_{i}\right)$ are equal for all $1 \leq i \leq d$. Furthermore, if $d \geq 2$ then $\chi_{1}\left(b_{i}\right)=\beta_{i i^{*} 0}$ for $0 \leq i \leq d$; that is, $\left(A, B, \chi_{1}\right)$ is standard.

Remark 1 (i) When $d=1$ under the hypotheses of the theorem, then $\chi_{1}\left(b_{1}\right)$ and $o(B)$ need not be integers. This case is discussed after the proof of the theorem in Section 3 .

(ii) When $d \geq 2$ under the hypotheses of the theorem, then $(A, B)$ is a commutative $\mathrm{C}$-algebra; that is, a commutative RBA with a standard degree map $\chi_{1}$. Then $B$ gives rise to a dual basis $\hat{B}$, where $\left\{m_{s}\right\}_{s=1}^{k}$ is the set of standard degrees for $\hat{B}$ $(k=d+1)$ and $o(B)=o(\hat{B})$ (see [4, Section II.5] or [5, Section 2]). So from Theorem $1,1+d \chi_{1}\left(b_{1}\right)=o(B)=1+d m_{2}$. Hence, the constant multiplicities equal the constant degrees.

Corollary 1 Suppose that $(A, B)$ is a fusion ring with $\delta=\chi_{1}$, the unique positive degree map. If the multiplicities $m_{s}$ are equal for $2 \leq s \leq k$ then $A$ is commutative and 
the degrees $\chi_{1}\left(b_{i}\right)$ are equal for $1 \leq i \leq d$. Also, if $d \geq 2$ then all degrees $\chi_{1}\left(b_{i}\right)=1$ and $B$ is an abelian group.

Corollary 2 Suppose that $(A, B)$ is the adjacency algebra of an association scheme, with $\delta=\chi_{1}$ the standard degree map. If the multiplicities $m_{s}$ are equal for all $2 \leq$ $s \leq k$ then $A$ is commutative and the valencies $\chi_{1}\left(b_{i}\right)$ are equal for all $1 \leq i \leq d$.

Some known results on RBAs are collected in Section 2. The proof of Theorem 1 is given in Section 3.

\section{Known results}

Some of the proofs that are not included in this section are found explicitly in [2]. Others are implicit in the proofs of special cases in [12], [4], [1], or [5]; and still others are straightforward from first principles. We retain the notation from Section 1. Throughout, $(A, B)$ is a RBA with a distinguished degree map $\delta$, anti-automorphism *, and basis $B=\left\{b_{0}=1_{A}, b_{1}, \ldots, b_{d}\right\}$; the irreducible characters of $A$ are denoted as $\delta=\chi_{1}, \chi_{2}, \ldots, \chi_{k}$ with $\chi_{s}(1)=n_{s} \in \mathbb{Z}_{>0}$ for $1 \leq s \leq k$; the corresponding primitive central idempotents are $e_{s}$ for $1 \leq s \leq k$; and $\tau=\sum_{s=1}^{k} m_{s} \chi_{s}$ is the standard feasible trace map, so that the $m_{s}$ are the multiplicities. The structure constants for $B$ are again denoted as $\beta_{i j l}, 0 \leq i, j, l \leq d$.

Proposition 1 For all $1 \leq s \leq k, m_{s}>0$.

Proof For all $x \in A$, with $x=\sum_{i=0}^{d} \alpha_{i} b_{i}$ for $\alpha_{i} \in \mathbb{C}$, define

$$
\bar{x}:=\sum_{i=0}^{d} \overline{\alpha_{i}} b_{i},
$$

where $\overline{\alpha_{i}}$ denotes the complex conjugate of $\alpha_{i}$. Then for all $x, y \in A, \overline{(x+y)}=\bar{x}+\bar{y}$; and since all the $\beta_{i j l}$ are real, $\overline{(x y)}=\bar{x} \bar{y}$. Hence,

$$
\left\{\overline{e_{s}} \mid 1 \leq s \leq k\right\}=\left\{e_{s} \mid 1 \leq s \leq k\right\}=\left\{\overline{e_{s}^{*}} \mid 1 \leq s \leq k\right\} .
$$

Also, $\tau\left(x \overline{x^{*}}\right)=o(B) \sum_{i=0}^{d} \alpha_{i} \overline{\alpha_{i}} \beta_{i i^{*} 0}>0$ for all $x \in A$ with $x \neq 0$. So $\tau\left(e_{s} \overline{e_{s}{ }^{*}}\right)>0$. Since either $\overline{e_{s}^{*}}=e_{s}$ or $e_{s} \overline{e_{s}^{*}}=0$, it follows that $\overline{e_{s}^{*}}=e_{s}$ and $\tau\left(e_{s}\right)>0$. But $\tau\left(e_{s}\right)=$ $\sum_{u=1}^{k} m_{u} \chi_{u}\left(e_{s}\right)=m_{s} n_{s}$. Hence, $m_{s}>0$.

Definition 8 If $\phi \in \operatorname{Hom}_{\mathbb{C}}(A, \mathbb{C})$ and $\sigma$ is a field automorphism of $\mathbb{C}$, the functional $\phi^{\sigma} \in \operatorname{Hom}_{\mathbb{C}}(A, \mathbb{C})$ is defined as the $\mathbb{C}$-linear map: $A \rightarrow \mathbb{C}$ such that $\phi^{\sigma}\left(b_{i}\right)=\phi\left(b_{i}\right)^{\sigma}$, for all $b_{i} \in B$.

Proposition 2 Suppose that $X: A \rightarrow M_{n}(\mathbb{C})$ is a representation of $A$ that affords the character $\chi$. Let $X\left(b_{i}\right)=\left(x_{j l}\left(b_{i}\right)\right)$, the $n \times n$ matrix with $j$, l entry $x_{j l}\left(b_{i}\right) \in \mathbb{C}$. Define $X^{\sigma}\left(b_{i}\right)=\left(\left(x_{j l}\left(b_{i}\right)\right)^{\sigma}\right)$, and extend $X^{\sigma} \mathbb{C}$-linearly to A. If $\beta_{i j l}^{\sigma}=\beta_{i j l}$ for all $0 \leq i, j, l \leq d$, then $X^{\sigma}$ is also a representation of $A$, and it affords the character $\chi^{\sigma}$. Furthermore, $\chi$ is irreducible if and only if $\chi^{\sigma}$ is irreducible. 
Corollary 3 Suppose that $\beta_{i j l} \in \mathbb{Q}, 0 \leq i, j, l \leq d$. Let $\sigma$ be a field automorphism of $\mathbb{C}$. Then the correspondence $\chi_{s} \mapsto \chi_{s}^{\sigma}, 1 \leq s \leq k$, is a permutation of the irreducible characters of $A$.

Definition 9 Define $\zeta$ as the sum of the irreducible characters of $A$; that is,

$$
\zeta:=\sum_{s=1}^{k} \chi_{s}
$$

Proposition 3 Suppose that $\beta_{i j l} \in \mathbb{Z}, 0 \leq i, j, l \leq d$. Then $\chi_{s}\left(b_{i}\right)$ is an algebraic integer for $0 \leq i \leq d$ and $1 \leq s \leq k$; and $\zeta\left(b_{i}\right) \in \mathbb{Z}$.

Proof The eigenvalues of each matrix $\left(\beta_{i j l}\right)$ (rows/columns indexed by $j / l$, for $0 \leq i \leq d)$ are algebraic integers. Since $A$ is semisimple, the (left) regular representation of $A$ with respect to $B$ is equivalent to a representation where each irreducible representation of $A$ (up to equivalence) appears as a diagonal block, and the entries below these blocks are zero. It follows that each $\chi_{s}\left(b_{i}\right)$ is an algebraic integer. That $\zeta\left(b_{i}\right) \in \mathbb{Z}$ follows from Corollary 3 .

Definition 10 The symmetric bilinear form $\langle$,$\rangle on \operatorname{Hom}_{\mathbb{C}}(A, \mathbb{C})$ is defined as follows: for all $\phi, \theta \in \operatorname{Hom}_{\mathbb{C}}(A, \mathbb{C})$,

$$
\langle\phi, \theta\rangle=\sum_{i=0}^{d} \frac{1}{\beta_{i i^{*} 0}} \phi\left(b_{i}\right) \theta\left(b_{i}^{*}\right) .
$$

Theorem 2 (Orthogonality Relations [12, (5.4)], [5, Proposition 2.11], [4, Theorem II.5.5]) For all $1 \leq s, t \leq k$,

$$
\left\langle\chi_{s}, \chi_{t}\right\rangle=\delta_{s t} o(B) \frac{n_{s}}{m_{s}} .
$$

Corollary 4 For all $1 \leq s \leq k, \overline{\chi_{s}}$ is an irreducible character of $A$, and $\overline{\chi_{s}}\left(b_{i}\right)=$ $\chi_{s}\left(b_{i}{ }^{*}\right)$ for all $0 \leq i \leq d$.

Proof The first statement follows from Proposition 2, since all $\beta_{i j l} \in \mathbb{R}$. If $X$ is an irreducible representation of $A$ that affords $\chi_{s}$, then

$$
b_{i} \longmapsto \operatorname{transpose}\left(X\left(b_{i}^{*}\right)\right)
$$

defines another irreducible representation, whose trace at $b_{i}$ is $\chi_{s}\left(b_{i}{ }^{*}\right)$. So there exists an irreducible character $\tilde{\chi}$ with $\tilde{\chi}\left(b_{i}\right)=\overline{\chi\left(b_{i}^{*}\right)}$ for all $0 \leq i \leq d$. By Definition 10, and since $\chi_{s}(1)=n_{s}>0$,

$$
\left\langle\chi_{s}, \tilde{\chi}\right\rangle=\sum_{i=0}^{d} \frac{1}{\beta_{i i^{*} 0}} \chi_{s}\left(b_{i}\right) \tilde{\chi}\left(b_{i}^{*}\right)=\sum_{i=0}^{d} \frac{1}{\beta_{i i^{*} 0}} \chi_{s}\left(b_{i}\right) \overline{\chi_{s}\left(b_{i}\right)}>0 .
$$

By Theorem $2, \overline{\chi\left(b_{i}^{*}\right)}=\chi\left(b_{i}\right)$ for all $i$. The second claim follows. 
Corollary 5 (i) If $\delta$ is any degree map then $\delta\left(b_{i}\right)=\delta\left(b_{i}{ }^{*}\right)$ for all $b_{i} \in B$. (ii) A has at most one positive degree map.

Corollary 6 If $\delta=\chi_{1}$ is any distinguished degree map, and if $o(B)$ is defined in terms of $\delta$, then $m_{1}=1$.

Proof By Theorem 2, $\left\langle\chi_{1}, \chi_{1}\right\rangle=o(B) \frac{n_{1}}{m_{1}}=o(B) / m_{1}$. But by Definition 10, Corollary 5(i) and Definition 3,

$$
\left\langle\chi_{1}, \chi_{1}\right\rangle=\sum_{i=0}^{d} \chi_{1}\left(b_{i}\right)^{2} / \beta_{i i^{*} 0}=o(B) .
$$

Theorem 3 ([1, Lemma 2.9], [2, Theorem 3.14]) If all $\beta_{i j l} \geq 0$ then A has a positive degree map.

\section{Proof of Theorem 1}

Let $(A, B)$ be a RBA with a full degree map $\chi_{1}$ that satisfies the hypotheses of Theorem 1 . The notation from the previous sections is in force. We assume that $d>0$ and hence $k>1$, as otherwise everything is trivial. Let $t$ denote the constant value $m_{s}$ for $2 \leq s \leq k$. Then $t>0$ by Proposition 1 . Now by Definition 7, Corollary 6, and Definition 9,

$$
\tau=\chi_{1}+t \sum_{s \geq 2} \chi_{s}=t \zeta+(1-t) \chi_{1} .
$$

Since $\tau\left(b_{i}\right)=0$ for all $b_{i} \neq b_{0}$, we have

$$
\zeta\left(b_{i}\right)=\left(1-\frac{1}{t}\right) \chi_{1}\left(b_{i}\right), \text { all } 1 \leq i \leq d .
$$

So by Proposition 3,

$$
\left(1-\frac{1}{t}\right) \chi_{1}\left(b_{i}\right) \in \mathbb{Z}, \text { all } 1 \leq i \leq d .
$$

Also from (1), we have

$$
o(B)=\tau(1)=\chi_{1}(1)+t \sum_{s \geq 2} \chi_{s}(1)=1+t \sum_{s \geq 2} n_{s} .
$$

By hypothesis, $\chi_{1}\left(b_{i}\right) \geq \beta_{i i^{*} 0} \in \mathbb{Z}_{>0}$ for $0 \leq i \leq d$. This and (4) yield

$$
\begin{aligned}
d+1 & \leq \sum_{i=0}^{d} \beta_{i i^{*} 0} \leq \sum_{i=0}^{d} \chi_{1}\left(b_{i}\right) \leq \sum_{i=0}^{d} \chi_{1}\left(b_{i}\right)^{2} / \beta_{i i^{*} 0}=o(B)=1+t \sum_{s \geq 2} n_{s} \\
& \leq 1+t \sum_{s \geq 2} n_{s}{ }^{2}=1+d t .
\end{aligned}
$$


If $t=1$, then all the inequalities of (5) must be equalities. In particular, $n_{s}{ }^{2}=n_{s}$ for all $s \geq 2$, so that $n_{s}=1$ for $1 \leq s \leq k$ and $A$ is commutative. Also, $\chi_{1}\left(b_{i}\right)=$ $\beta_{i i^{*} 0}=1$ for all $i$, so that $\left(A, B, \chi_{1}\right)$ is standard and the conclusion of Theorem 1 holds. Thus we may assume henceforth that $t \neq 1$.

For any field automorphism $\sigma$ of $\mathbb{C}$, each $\chi_{s}{ }^{\sigma}$ is an irreducible character of $A$, by Proposition 2, and

$$
\tau^{\sigma}=\chi_{1}^{\sigma}+t^{\sigma} \sum_{s \geq 2} \chi_{s}^{\sigma} .
$$

Since $\tau^{\sigma}\left(b_{i}\right)=\tau\left(b_{i}\right)^{\sigma}=0$ for $b_{i} \neq b_{0}$, and $\tau^{\sigma}\left(b_{0}\right)=o(B)^{\sigma}$, we have

$$
\tau^{\sigma}=\frac{o(B)^{\sigma}}{o(B)} \tau=\frac{o(B)^{\sigma}}{o(B)} \chi_{1}+\frac{o(B)^{\sigma}}{o(B)} t \sum_{s \geq 2} \chi_{s} .
$$

So if $k>2$, then (6), (7), $t \neq 1$, and linear independence of the $\chi_{s}$ imply that $\chi_{1}^{\sigma}=\frac{o(B)^{\sigma}}{o(B)} \chi_{1}$, hence $\chi_{1}^{\sigma}=\chi_{1}$ and $o(B)^{\sigma}=o(B)$ for all automorphisms $\sigma$ of $\mathbb{C}$. Thus if $k>2$, then $\chi_{1}\left(b_{i}\right) \in \mathbb{Q}$ for $0 \leq i \leq d$. Since $\chi_{1}\left(b_{i}\right)$ is an algebraic integer (Proposition 3), it follows that $\chi_{1}\left(b_{i}\right) \in \mathbb{Z}$ for $0 \leq i \leq d$, and $o(B) \in \mathbb{Q}$. Similarly, if $k=2$ but $\chi_{1}^{\sigma}=\chi_{1}$ for all automorphisms $\sigma$ of $\mathbb{C}$, then $\chi_{1}\left(b_{i}\right) \in \mathbb{Z}$ for all $i$. If $k=2$ and $\chi_{1}^{\sigma}=\chi_{2} \neq \chi_{1}$ for some $\sigma$ then $n_{2}=n_{1}=1$ implies that $A$ is commutative, and $d+1=k=2$.

So it suffices to assume for the rest of the proof that $t \neq 1$ and $\chi_{1}\left(b_{i}\right) \in \mathbb{Z}$ for $0 \leq i \leq d$. Then by (3),

$$
\frac{1}{t} \chi_{1}\left(b_{i}\right) \in \mathbb{Z}_{>0}, \quad \text { all } 1 \leq i \leq d
$$

By (4), $t=(o(B)-1) / \sum_{s \geq 2} n_{s}$. Then (8) yields

$$
\left(\sum_{s \geq 2} n_{s}\right) \cdot \chi_{1}\left(b_{i}\right) /(o(B)-1) \in \mathbb{Z}_{>0}, \quad \text { all } 1 \leq i \leq d,
$$

so that

$$
o(B)-1 \leq\left(\sum_{s \geq 2} n_{s}\right) \cdot \chi_{1}\left(b_{i}\right), \quad \text { all } 1 \leq i \leq d .
$$

Let $\beta=\min _{i>0} \chi_{1}\left(b_{i}\right)$. Then (9), in combination with part of (5), gives us

$$
\begin{aligned}
d \beta & \leq \sum_{i=1}^{d} \chi_{1}\left(b_{i}\right) \leq \sum_{i=1}^{d} \chi_{1}\left(b_{i}\right)^{2} / \beta_{i i^{*} 0}=o(B)-1 \leq\left(\sum_{s \geq 2} n_{s}\right) \cdot \beta \\
& \leq\left(\sum_{s \geq 2} n_{s}{ }^{2}\right) \cdot \beta=d \beta .
\end{aligned}
$$

So all the inequalities of (10) are equalities. Then $\sum_{s \geq 2} n_{s}=\sum_{s \geq 2} n_{s}{ }^{2}$ implies that all $n_{s}=1$ and $A$ is commutative. Also, $d \beta=\sum_{i=1}^{d} \chi_{1}\left(b_{i}\right)=\sum_{i=1}^{d} \chi_{1}\left(b_{i}\right)^{2} / \beta_{i i^{*} 0}$, 
with $\beta \leq \chi_{1}\left(b_{i}\right) \leq \chi_{1}\left(b_{i}\right)^{2} / \beta_{i i^{*} 0}$, yields that $\beta=\chi_{1}\left(b_{i}\right)=\beta_{i i^{*} 0}$, all $1 \leq i \leq d$. The proof is complete.

Remark 2 Suppose that $(A, B)$ is a RBA with integer structure constants and $d=1$. Then $B=\left\{b_{0}, b_{1}\right\}$ and $b_{1}^{2}=\beta_{110} b_{0}+\beta_{111} b_{1}$. So $A$ has two irreducible characters $\chi_{1}$ and $\chi_{2}$, with $\chi_{1}\left(b_{0}\right)=\chi_{2}\left(b_{0}\right)=1$. Furthermore, $\chi_{1}\left(b_{1}\right)$ and $\chi_{2}\left(b_{1}\right)$ are the roots of the polynomial $x^{2}-\beta_{111} x-\beta_{110}=0$, namely $\left(\beta_{111} \pm \sqrt{\beta_{111}^{2}+4 \beta_{110}}\right) / 2$. This RBA exists for any choice of $\beta_{110} \in \mathbb{Z}_{>0}$ and $\beta_{111} \in \mathbb{Z}$. Let $\chi_{1}\left(b_{1}\right)=\left(\beta_{111}+\right.$ $\left.\sqrt{\beta_{111^{2}+4 \beta_{110}}}\right) / 2$. Then $\chi_{1}$ is a full degree map if and only if $\beta_{111} \geq \beta_{110}-1$. But $\chi_{1}\left(b_{1}\right)$ is not necessarily an integer, much less necessarily equal to $\beta_{110}$. Finally, $(A, B)$ is a fusion ring if and only if $\beta_{110}=1$ and $\beta_{111} \in \mathbb{Z}_{>0}$.

\section{References}

1. Arad, Z., Blau, H.I.: On table algebras and applications to finite group theory. J. Algebra 138, 137-185 (1991)

2. Arad, Z., Fisman, E., Muzychuk, M.: Generalized table algebras. Israel J. Math. 114, 29-60 (1999)

3. Bagherian, J., Barghi, A.R.: Standard character condition for C-algebras, arXiv:0803.2423 [Math. RT] (17 March 2008)

4. Bannai, E., Ito, T.: Algebraic Combinatorics I. Association Schemes. Benjamin/Cummings, Menlo Park (1984)

5. Blau, H.I.: Quotient structures in C-algebras. J. Algebra 175, 24-64 (1995)

6. Blau, H.I.: Table algebras. European J. Combin. 30, 1426-1455 (2009)

7. Blau, H.I., Zieschang, P.-H.: Sylow theory for table algebras, fusion rule algebras, and hypergroups. J. Algebra 273, 551-570 (2004)

8. Evdokimov, S.A., Ponomorenko, I.N., Vershik, A.M.: Algebras in Plancherel duality and algebraic combinatorics. Funct. Anal. Appl. 31, 252-261 (1997)

9. Gelaki, S., Nikshych, D.: Nilpotent fusion categories. Adv. Math. 217, 1053-1071 (2008)

10. Green, R.M.: Tabular algebras and their asymptotic versions. J. Algebra 252, 27-64 (2002)

11. Hanaki, A., Uno, K.: Algebraic structure of association schemes of prime order. J. Algebraic Combin. 23, 189-195 (2006)

12. Higman, D.G.: Coherent algebras. Linear Algebra Appl. 93, 209-239 (1987)

13. Kawada, Y.: Über den Dualitätssatz der Charaktere nicht commutativen Gruppen. Proc. Phys. Math. Soc. Japan 24(3), 97-109 (1942)

14. Sunder, V.S., Wildberger, N.J.: Actions of finite hypergroups. J. Algebraic Combin. 18, 135-151 (2003)

15. Zieschang, P.-H.: An Algebraic Approach to Association Schemes. Lecture Notes in Mathematics, vol. 1628. Springer, Berlin (1996) 